\title{
Decreased Aortic Elasticity in Children With Marfan Syndrome or Loeys-Dietz Syndrome
}

\author{
Yohei Akazawa, MD, PhD; Noriko Motoki, MD, PhD; Akira Tada, MD; Shoko Yamazaki, MD; \\ Akira Hachiya, MD; Satoshi Matsuzaki, MD, PhD; Motoko Kamiya, MD; \\ Tomohiko Nakamura, MD, PhD; Tomoki Kosho, MD, PhD; Yuji Inaba, MD, PhD
}

\begin{abstract}
Background: The characteristics of aortic elasticity are unclear in children with connective tissue disorders (CTDs) such as Marfan syndrome (MFS) and Loeys-Dietz syndrome (LDS), especially in those with a non-dilated aortic root (AoR). This study evaluated the aortic elasticity properties of pediatric MFS and LDS patients with either dilated or non-dilated AoR.
\end{abstract}

\begin{abstract}
Methods and Results: The 31 children with MFS or LDS were classified into dilated (Z score of AoR diameter $\geq 2.5$; $\mathrm{n}=17$ ) or non-dilated ( $\mathrm{Z}$ score of AoR diameter <2.5; $\mathrm{n}=14$ ) AoR groups and compared with controls. Using transthoracic echocardiography, we analyzed the aortic elasticity parameters of distensibility, strain, and stiffness index at the levels of the AoR, sinotubular junction, ascending aorta, and descending aorta. Aortic distensibility and strain were significantly lower in both test groups than in controls at the AoR level. The $Z$ score of AoR diameter significantly correlated with aortic distensibility $(R=-0.63, P<0.001)$, strain $(R=-0.54, P=0.002)$, and stiffness index $(R=0.52, P=0.002)$ in the patients' groups. Multivariate analysis revealed that aortic distensibility and the type of $C T D$ were independently associated with AoR dilatation.
\end{abstract}

Conclusions: Aortic elasticity at the level of the AoR may be decreased in children with MFS or LDS even before AoR dilatation progresses. Less aortic distensibility and CTD type are considered important parameters in estimating AoR dilatation in these patients. (Circ $J$ 2016; 80: 2369-2375)

Key Words: Aortic elasticity; Connective tissue disorders

A ortic root (AoR) dilatation and dissection are the main factors affecting prognosis in patients with connective tissue disorders (CTDs) such as Marfan syndrome (MFS) and Loeys-Dietz syndrome (LDS). ${ }^{1,2}$ Although aortic diameter has been the main predictor of aortic complications, ${ }^{3}$ aortic dissection can nonetheless occur in patients with AoR diameters below the indication for surgical intervention. ${ }^{4}$ Thus, the assessment of aortic diameter alone may not be sufficient to prospect clinical outcome. Aortic wall pathogenesis among the CTDs is similar, and includes fragmentation of elastic fibers and collagen deposition, ${ }^{5}$ leading to aortic stiffness. ${ }^{6,7}$ Nollen et al demonstrated that aortic stiffness could also be an independent predictor of progressive aortic dilatation in adult MFS patients. ${ }^{8}$ Increased aortic stiffness has been reported in children and adults with MFS, LDS, and other CTDs. ${ }^{9-11}$ However, the aortic elasticity properties in children with MFS or LDS have not been studied in detail, especially in patients without AoR dilatation.
The present study evaluated the elasticity of the aorta, including distensibility, strain, and stiffness index, using transthoracic echocardiography (TTE) in children with MFS and LDS with and without AoR dilatation.

\section{Methods}

This investigation was a retrospective review of patients' clinical data at Shinshu University School of Medicine. The study protocol was approved by the institution's Ethics Committee, and written informed consent was given by the parents of the children in the patient and controls groups enrolled in the study.

\section{Subjects}

Children with MFS or LDS who were followed as outpatients between 2007 and 2016 at Shinshu University Hospital were divided into 2 groups according to AoR diameter: the dilated group was defined as patients having an AoR diameter Z score

Received July 24, 2016; revised manuscript received September 4, 2016; accepted September 21, 2016; released online October 13, 2016 Time for primary review: 29 days

Department of Pediatrics (Y.A., N.M., A.T., S.Y., A.H., S.M., M.K., Y.I.), Department of Medical Genetics (M.K., T.K.), Division of Neonatology (T.N.), Shinshu University School of Medicine, Matsumoto; Division of Neonatology, Nagano Children's Hospital, Azumino (T.N.), Japan

Mailing address: Yohei Akazawa, MD, PhD, Department of Pediatrics, Shinshu University School of Medicine, 3-1-1 Asahi, Matsumoto 390-8621, Japan. E-mail: yohei@ck9.so-net.ne.jp

ISSN-1346-9843 doi:10.1253/circj.CJ-16-0739

All rights are reserved to the Japanese Circulation Society. For permissions, please e-mail: cj@j-circ.or.jp 


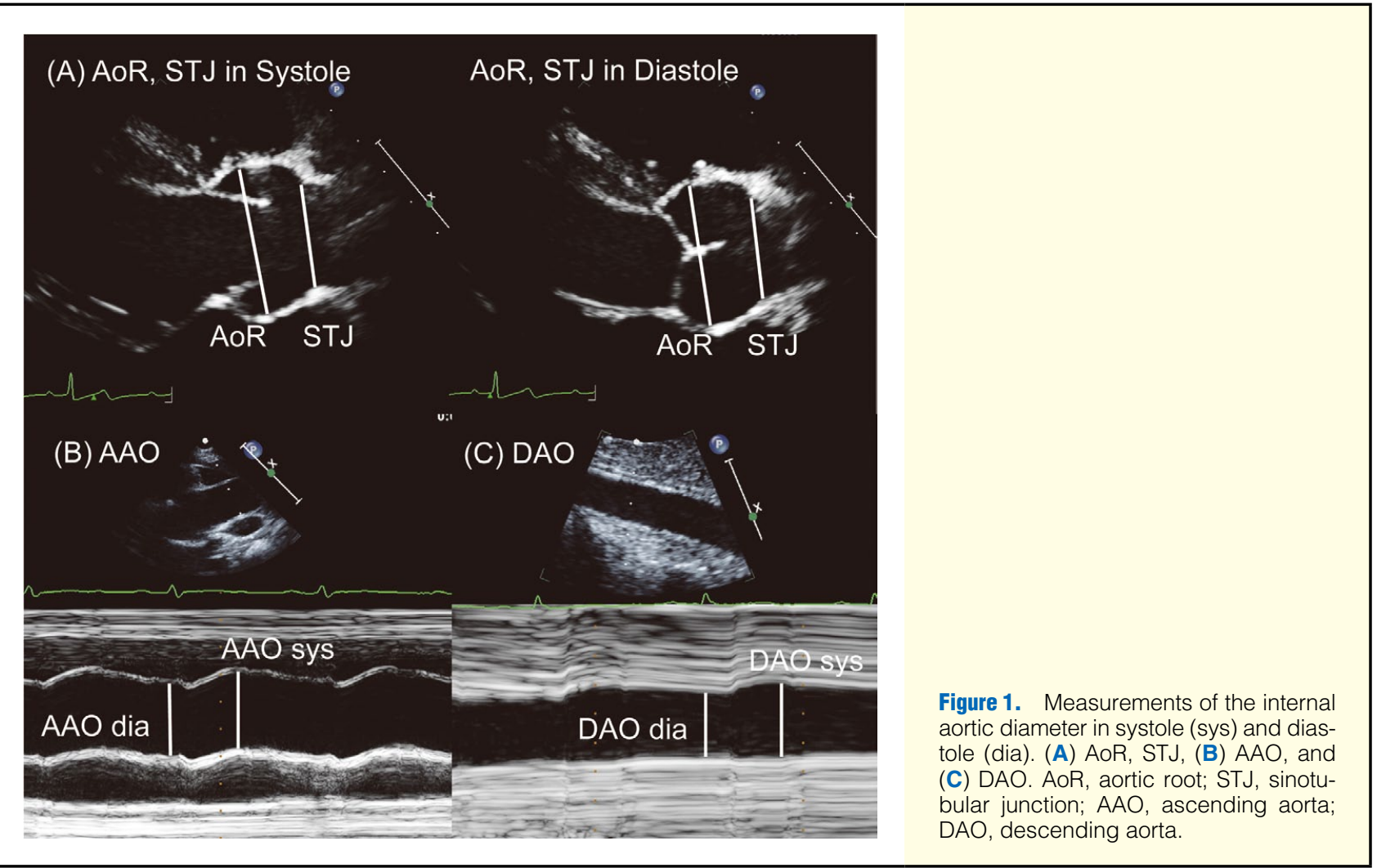

$>2.5^{12}(n=17)$, and the non-dilated group contained patients with an AoR diameter $\mathrm{Z}$ score $<2.5(\mathrm{n}=14)$. Demographic and clinical data were obtained from medical records. The revised Ghent nosology was used for the diagnosis of MFS. ${ }^{13}$ All MFS patients harbored a fibrillin-1 (FBNI) mutation, while all LDS patients exhibited transforming growth factor- $\beta$ receptor 2 (TGFBR 2) or SMAD 3 mutations and the clinical characteristics of LDS. We enrolled 15 subjects as controls; they presented with signs of heart disease or arrhythmia that were subsequently identified by further examination. None of the control subjects had a prior or family history of systemic disease.

\section{Measurement of Aortic Elastic Properties}

A trained physician (Y.A.) performed all TTE examinations with an iE33 device (Philips Healthcare, Andover, MA, USA). The diameters of the AoR and sinotubular junction (STJ) were measured at mid-systole and end-diastole using the inner-toinner edge method in a 2D parasternal long-axis view according to the guidelines of the American Society of Echocardiography $^{14}$ (Figure 1A). The diameter of the proximal ascending aorta (AAO) was measured at $2-3 \mathrm{~cm}$ from the valve at endsystole and end-diastole using the leading edge to leading edge principle in $\mathrm{M}$-mode ${ }^{15}$ (Figure 1B). The diameter of the descending aorta (DAO) was measured in a subcostal long-axis view at the level of the diaphragm ${ }^{14}$ (Figure 1C). All echocardiographic measurements were averaged from 3 consecutive heartbeats. The $\mathrm{Z}$ scores for the AoR, STJ, AAO, and DAO were calculated using normative data. ${ }^{16,17}$ Systolic and diastolic blood pressures (SBP and DBP, respectively) were measured in the right arm by sphygmomanometer (ES-H55, Terumo Co, Tokyo, Japan) with the patient in a supine position. The mean of 3 measurements were averaged for ensuing calculations. The following 3 parameters of aortic stiffness were calculated at the levels of the AoR, STJ, AAO, and DAO: ${ }^{15,18,19}$

Aortic distensibility $=2 \times(\mathrm{AOs}-\mathrm{AOd}) /(\mathrm{AOd}) \times(\mathrm{SBP}-\mathrm{DBP})$

Aortic strain $(\%)=100 \times($ AOs - AOd $) / A O d$

Aortic stiffness index $(\beta)=\ln (\mathrm{SBP} / \mathrm{DBP}) /([\mathrm{AOs}-\mathrm{AOd}] / \mathrm{AOd})$

Where AOs and AOd are the systolic and diastolic aortic dimensions, respectively, and $\mathrm{ln}$ is the natural logarithm.

\section{Statistical Analysis}

Data are expressed as the mean \pm standard deviation (SD). In group comparisons, Student's unpaired t-test was used for parametric variables and the Mann-Whitney U test was adopted for nonparametric variables. Categorical variables were analyzed using the Chi-square test. For comparisons of clinical characteristics and echocardiography parameters among the 3 groups, one-way analysis of variance and the post hoc Bonferroni test were performed. Spearman's correlation analysis was used to assess for relationships between the $\mathrm{Z}$ scores of the aortic segments and aortic elasticity indexes. Multivariate regression analysis was also performed to determine the independent effect of aortic distensibility on aortic dilatation using independent variables potentially influencing aortic dilatation. Four factors (aortic distensibility, type of CTD [MFS was coded as 1 and LDS was coded as 0], age, SBP and DBP) were then entered into a multiple linear regression model. We chose the distensibility of the AoR because of its better correlation with AoR dilatation compared with both aortic strain and aortic stiffness index using univariate regression analysis. We chose other factors, including age, SBP, and DBP, that could be influencing factors for aortic dilatation and stiffness. ${ }^{20,21}$ Intraobserver variability was assessed by one investigator (Y.A.) conducting off-line measurements on the same patients 4 weeks apart. Interobserver variability was assessed 


\begin{tabular}{|c|c|c|c|c|c|c|c|c|}
\hline \multirow[b]{2}{*}{ Variable } & \multirow{2}{*}{$\begin{array}{c}\text { Controls } \\
(n=15)\end{array}$} & \multirow{2}{*}{$\begin{array}{c}\text { Total } \\
\text { patients } \\
(n=31)\end{array}$} & \multirow{2}{*}{$\begin{array}{c}\text { AoR-ND } \\
(n=14)\end{array}$} & \multirow{2}{*}{$\begin{array}{l}\text { AoR-D } \\
(n=17)\end{array}$} & \multicolumn{4}{|c|}{$P$ value } \\
\hline & & & & & $\begin{array}{c}\text { Control vs. } \\
\text { overall }\end{array}$ & $\begin{array}{c}\text { Control vs. } \\
\text { AoR-D }\end{array}$ & $\begin{array}{c}\text { Control vs. } \\
\text { AoR-ND }\end{array}$ & $\begin{array}{c}\text { AoR-D vs. } \\
\text { AoR-ND }\end{array}$ \\
\hline Age (years) & $8.9 \pm 6.1$ & $12.1 \pm 6.7$ & $10.6 \pm 7.2$ & $13.3 \pm 6.1$ & NS & NS & NS & NS \\
\hline$<18(n)$ & 13 & 26 & 11 & 14 & NS & NS & NS & NS \\
\hline Sex (M/F) & $6 / 9$ & $15 / 16$ & $5 / 9$ & $10 / 7$ & NS & NS & NS & NS \\
\hline \multicolumn{9}{|l|}{ Type of CTD (n) } \\
\hline MFS & & 26 & 14 & 12 & & & & $<0.05$ \\
\hline LDS & & 5 & 0 & 5 & & & & NS \\
\hline Body surface area $\left(\mathrm{m}^{2}\right)$ & $1.0 \pm 0.5$ & $1.3 \pm 0.5$ & $1.2 \pm 0.5$ & $1.4 \pm 0.5$ & NS & NS & NS & NS \\
\hline $\mathrm{SBP}(\mathrm{mmHg})$ & $100.2 \pm 11.2$ & $99.7 \pm 15.0$ & $96.7 \pm 16.3$ & $102.0 \pm 14.0$ & NS & NS & NS & NS \\
\hline $\mathrm{DBP}(\mathrm{mmHg})$ & $54.1 \pm 8.8$ & $53.4 \pm 10.9$ & $52.2 \pm 9.8$ & $54.4 \pm 11.8$ & NS & NS & NS & NS \\
\hline Pulse pressure (mmHg) & $46.1 \pm 8.0$ & $45.8 \pm 10.6$ & $43.7 \pm 10.7$ & $47.6 \pm 10.5$ & NS & NS & NS & NS \\
\hline Heart rate (beats/min) & $75.1 \pm 14.1$ & $71.8 \pm 19.0$ & $67.8 \pm 17.6$ & $74.0 \pm 17.4$ & NS & NS & NS & NS \\
\hline \multicolumn{9}{|l|}{ Medication (n) } \\
\hline$\beta$-blocker only & 0 & 4 & 2 & 2 & & & & NS \\
\hline$\beta$-blocker and ARB & 0 & 20 & 7 & 13 & & & & NS \\
\hline No medication & 15 & 7 & 5 & 2 & & & & NS \\
\hline
\end{tabular}

Data are presented as mean \pm standard deviation or count (\%). AoR, aortic root; AoR-ND, aortic root non-dilated group; AoR-D, aortic root dilated group; MFS, Marfan syndrome; LDS, Loeys-Dietz syndrome; CTD, connective tissue disorder; SBP, systolic blood pressure; DBP, diastolic blood pressure; ARB, angiotensin-receptor blocker.

\begin{tabular}{|c|c|c|c|c|c|c|}
\hline & & & & & $P$ value & \\
\hline Variable & $(n=15)$ & $(n=14)$ & $(n=17)$ & $\begin{array}{c}\text { Control vs. } \\
\text { AoR-D }\end{array}$ & $\begin{array}{l}\text { Control vs. } \\
\text { AoR-ND }\end{array}$ & $\begin{array}{l}\text { AoR-D vs. } \\
\text { AoR-ND }\end{array}$ \\
\hline \multicolumn{7}{|l|}{ AoR } \\
\hline Diameter & $22.0 \pm 5.8$ & $26.6 \pm 5.7$ & $35.5 \pm 8.6$ & $<0.01$ & NS & $<0.01$ \\
\hline Diameter Z score & $0.66 \pm 0.83$ & $1.9 \pm 0.62$ & $3.8 \pm 1.2$ & $<0.01$ & $<0.01$ & $<0.01$ \\
\hline Distensibility $\left(\times 10^{-3} \mathrm{mmHg}^{-1}\right)$ & $2.4 \pm 1.1$ & $1.5 \pm 0.9$ & $0.5 \pm 0.3$ & $<0.01$ & $<0.05$ & $<0.01$ \\
\hline Strain $(\%)$ & $10.4 \pm 3.8$ & $5.1 \pm 2.6$ & $2.5 \pm 1.8$ & $<0.01$ & $<0.01$ & NS \\
\hline$\beta$-stifffness index & $0.03 \pm 0.02$ & $0.08 \pm 0.06$ & $0.3 \pm 0.3$ & $<0.01$ & NS & NS \\
\hline \multicolumn{7}{|l|}{ STJ } \\
\hline Diameter & $16.9 \pm 4.8$ & $20.3 \pm 4.6$ & $25.5 \pm 6.4$ & $<0.01$ & NS & $<0.05$ \\
\hline Diameter Z score & $0.20 \pm 0.70$ & $1.2 \pm 0.5$ & $2.3 \pm 1.1$ & $<0.01$ & $<0.05$ & $<0.01$ \\
\hline Distensibility $\left(\times 10^{-3} \mathrm{mmHg}^{-1}\right)$ & $3.7 \pm 1.6$ & $1.8 \pm 1.3$ & $1.4 \pm 0.8$ & $<0.01$ & $<0.01$ & NS \\
\hline Strain $(\%)$ & $16.0 \pm 6.0$ & $7.5 \pm 5.3$ & $6.3 \pm 3.8$ & $<0.01$ & $<0.01$ & NS \\
\hline$\beta$-stifffness index & $0.02 \pm 0.02$ & $0.08 \pm 0.08$ & $0.08 \pm 0.1$ & NS & NS & NS \\
\hline \multicolumn{7}{|l|}{ AAO } \\
\hline Diameter & $18.2 \pm 5.6$ & $20.7 \pm 4.4$ & $24.2 \pm 5.5$ & $<0.01$ & NS & NS \\
\hline Diameter Z score & $0.18 \pm 2.0$ & $-0.3 \pm 1.1$ & $0.8 \pm 1.3$ & NS & NS & NS \\
\hline Distensibility $\left(\times 10^{-3} \mathrm{mmHg}^{-1}\right)$ & $4.9 \pm 3.1$ & $2.9 \pm 1.8$ & $1.3 \pm 1.0$ & $<0.01$ & NS & NS \\
\hline Strain $(\%)$ & $21.5 \pm 12.4$ & $11.6 \pm 7.3$ & $6.0 \pm 4.6$ & $<0.01$ & $<0.05$ & NS \\
\hline$\beta$-stifffness index & $0.02 \pm 0.01$ & $0.04 \pm 0.04$ & $0.1 \pm 0.1$ & $<0.05$ & NS & NS \\
\hline \multicolumn{7}{|l|}{ DAO } \\
\hline Diameter & $11.6 \pm 3.3$ & $13.8 \pm 4.0$ & $15.2 \pm 2.7$ & $<0.05$ & NS & NS \\
\hline Diameter Z score & $0.034 \pm 1.5$ & $0.8 \pm 0.9$ & $1.1 \pm 0.7$ & $<0.05$ & NS & NS \\
\hline Distensibility $\left(\times 10^{-3} \mathrm{mmHg}^{-1}\right)$ & $4.4 \pm 1.4$ & $3.9 \pm 2.0$ & $3.1 \pm 1.9$ & NS & NS & NS \\
\hline Strain $(\%)$ & $19.6 \pm 4.6$ & $16.2 \pm 7.9$ & $14.2 \pm 7.9$ & NS & NS & NS \\
\hline$\beta$-stifffness index & $0.02 \pm 0.01$ & $0.03 \pm 0.02$ & $0.03 \pm 0.02$ & NS & NS & NS \\
\hline
\end{tabular}

Data are presented as mean \pm standard deviation or count (\%). STJ, sinotubular junction; AAO, ascending aorta; DAO, descending aorta. Other abbreviations as in Table 1.

by a second investigator (N.M.) who was unaware of the previous results and performed the same measurements on 10 randomly selected study participants. Intra- and interobserver agreements were assessed using the intraclass correlation coefficient (ICC). Additionally, agreement between investigators was tested using Bland-Altman analysis by calculating the bias (mean difference) and 1.96 SD around the mean difference. A P value $<0.05$ was considered statistically significant. All 

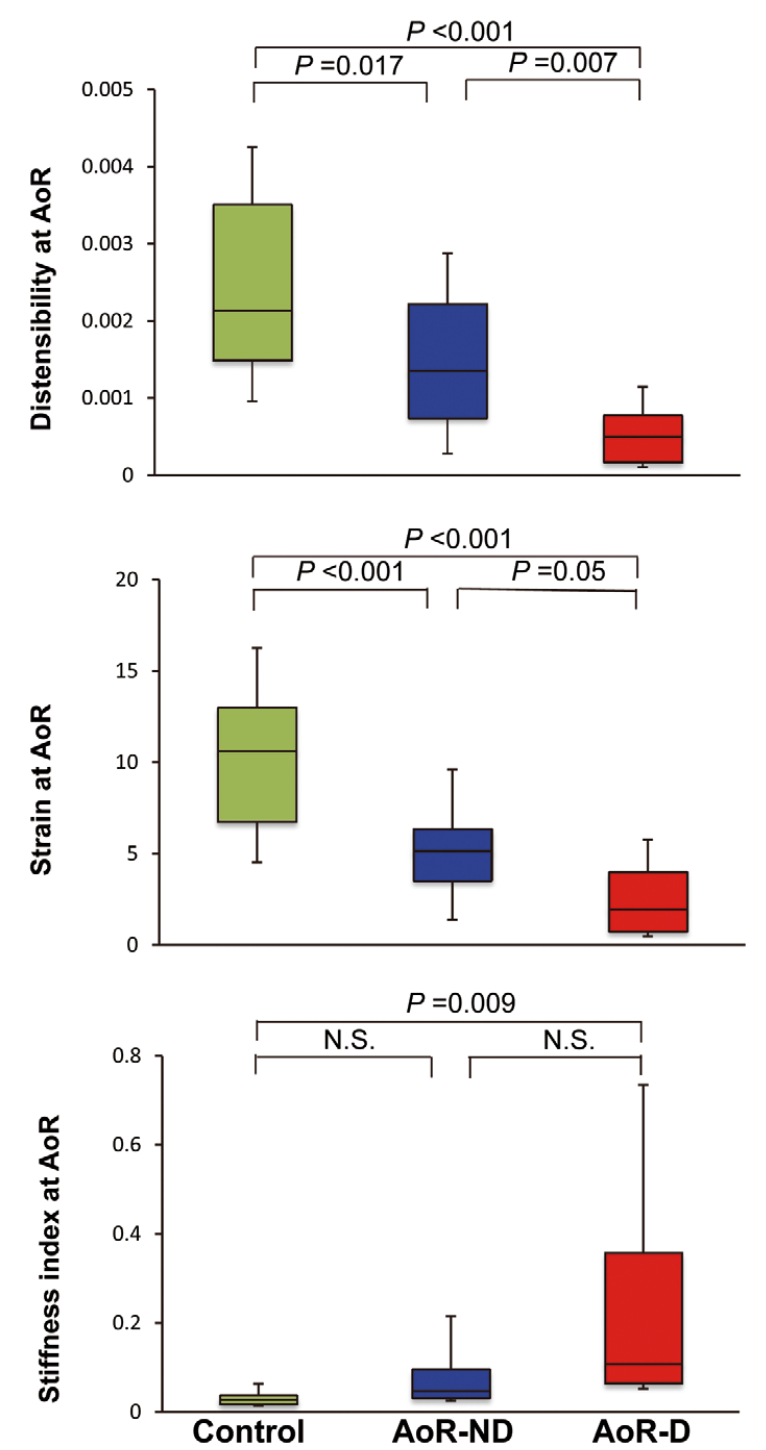

Figure 2. Box-and-whisker plots showing aortic distensibility, aortic strain, and aortic stiffness index in aortic root dilated group (AoR-D), aortic root non-dilated group (AoR-ND), and control subjects. The horizontal line in the middle of each box indicates the median, the top and bottom borders of each box show the 75th and 25th percentiles, respectively, and the whiskers mark the 90th and 10th percentiles. NS, not significant.

statistical analyses were carried out using SPSS version 23.0 software (SPSS, Inc, Chicago, IL, USA).

\section{Results}

\section{Study Subjects' Characteristics}

Table 1 shows the clinical characteristics of the 3 test groups, which were comparable with regard to age, sex, SBP, and DBP. CTD patients included 26 with MFS and 5 with LDS, and all were predominantly children $(<18$ years old; $84 \%)$. There were no LDS patients in the non-dilated group. Most CTD patients were receiving either $\beta$-blocker (atenolol) monotherapy or combination treatment using a $\beta$-blocker and angiotensin-receptor blocker (losartan). No significant difference was

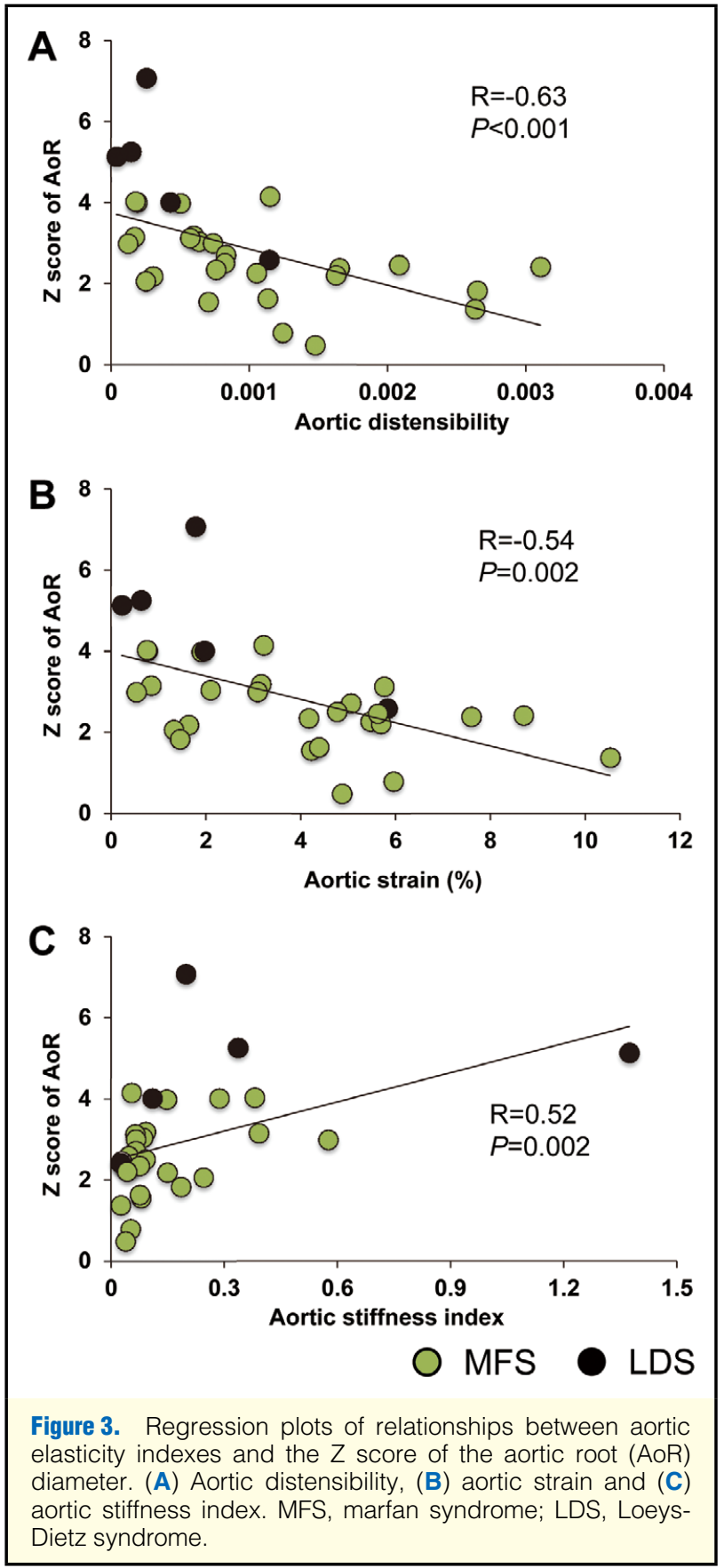



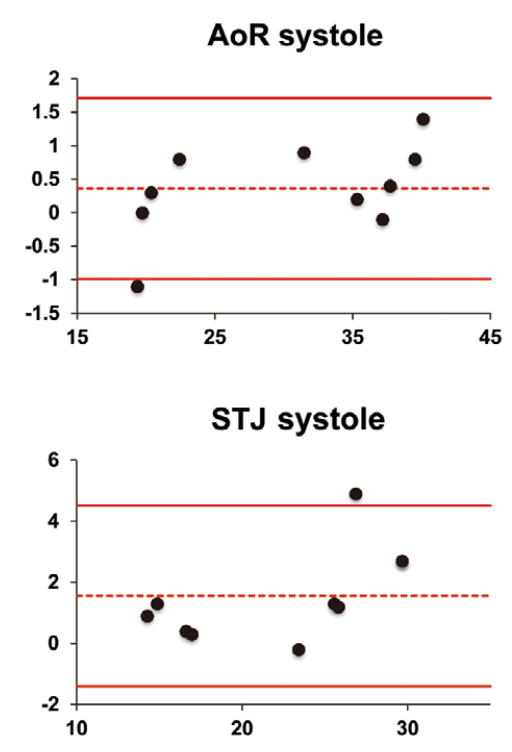

AAO systole

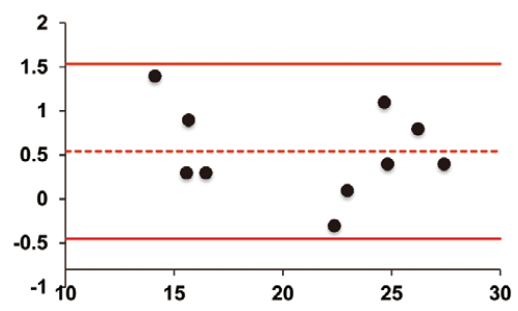

DAO systole

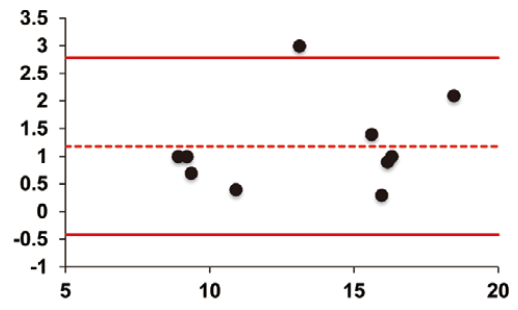

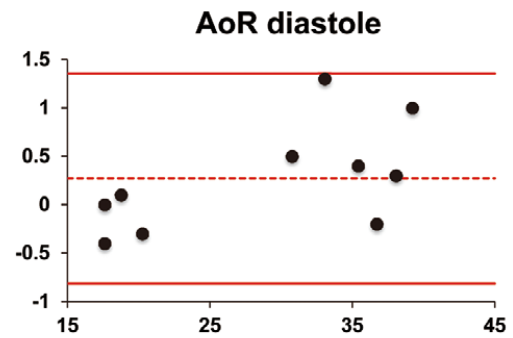

STJ diastole

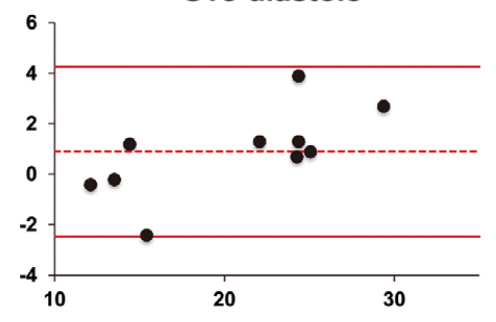

AAO diastole

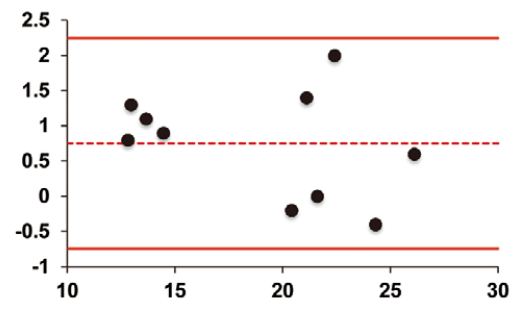

DAO diastole

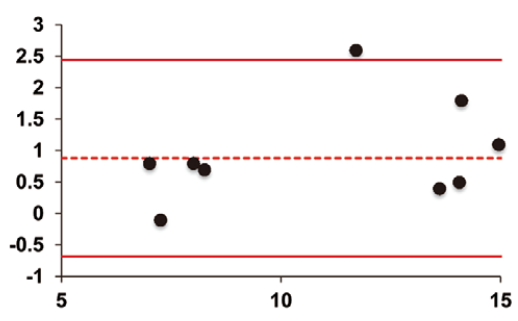

Figure 4. Bland-Altman plots of interobserver variability showing differences in the values for the AoR in systole and diastole, STJ in systole and diastole, AAO in systole and diastole, and DAO in systole and diastole. X-axis: absolute average of 2 measurements for each index. Y-axis: difference between the 2 measurements for each index. The dotted line shows the mean difference and the solid lines indicate the mean $\pm 1.96 \mathrm{SD}$. AoR, aortic root; STJ, sinotubular junction; $A A O$, ascending aorta; $\mathrm{DAO}$, descending aorta. observed between the dilated and non-dilated groups for use of medication. No subject had moderate or severe aortic regurgitation.

\section{Aortic Dimensions and Elastic Properties}

The data on aortic dimensions and stiffness at the levels of the AoR, STJ, AAO, and DAO are presented in Table 2. Both aortic distensibility and strain in the dilated group at all levels of the aorta apart from the DAO were significantly lower than in controls. The aortic stiffness index at the AoR and AAO levels in the dilated group was higher than in controls. Importantly, even in non-dilated patients, aortic distensibility, as well as strain, at the levels of the AoR and STJ was lower than in controls. At the AAO level in the non-dilated group, aortic strain was also lower as compared with controls (Table 2, Figure 2). Figure 3 presents the correlations between aortic elasticity parameters and the $\mathrm{Z}$ score of AoR diameter. Both aortic distensibility and aortic strain were negatively correlated
$(\mathrm{R}=-0.63, \mathrm{P}<0.001$ and $\mathrm{R}=-0.54, \mathrm{P}=0.002$, respectively), and the aortic stiffness index positively correlated $(\mathrm{R}=0.52, \mathrm{P}=0.002)$, with the AoR diameter $\mathrm{Z}$ score. These results indicated that lower aortic elasticity parameters at the AoR level correlated with aortic dilatation in young MFS and LDS patients not only in the dilated group, but also in the non-dilated group. At the AAO level, aortic strain was the only aortic elasticity index associated with the $\mathrm{Z}$ score of the AAO $(\mathrm{R}=-0.38)$. There were no significant correlations between the aortic elasticity indexes and aortic dilatation at the STJ and DAO levels. Multivariate regression analysis was conducted to identify the independent relevance of AoR dilatation in the patients' groups. Calculations using aortic distensibility, type of CTD, age, SBP, and DBP as independent variables revealed that aortic distensibility and CTD type (larger Z score of the AoR in the LDS group than in the MFS group) were significantly correlated with the $\mathrm{Z}$ score of the AoR (Table 3). 


\begin{tabular}{|lcccc|}
\hline \multicolumn{1}{c}{ Table 4. Reproducibility of Aortic Measurements in Pediatric Patients With MFS or LDS } & Intraobserver \\
Variable & $\begin{array}{c}\text { Interobserver } \\
\text { variability }\end{array}$ & ICC & $-0.26 \pm 1.2$ & ICC \\
AoR sys & $0.36 \pm 1.3$ & 0.99 & $0.27 \pm 1.6$ & 0.99 \\
AoR dia & $0.27 \pm 1.1$ & 0.99 & $-0.54 \pm 1.8$ & 0.99 \\
STJ sys & $1.6 \pm 3.0$ & 0.94 & $-0.47 \pm 3.0$ & 0.98 \\
STJ dia & $0.90 \pm 3.4$ & 0.95 & $-0.04 \pm 2.2$ & 0.97 \\
AAO sys & $0.54 \pm 1.0$ & 0.99 & $0.00 \pm 3.5$ & 0.97 \\
AAO dia & $0.75 \pm 1.5$ & 0.98 & $0.24 \pm 2.3$ & 0.93 \\
DAO sys & $1.2 \pm 1.6$ & 0.93 & $-0.11 \pm 3.8$ & 0.95 \\
DAO dia & $0.88 \pm 1.6$ & 0.95 & 0.82 \\
\hline
\end{tabular}

Data are presented as mean \pm standard deviation. AoR sys/dia, AoR diameter in systole/diastole; STJ sys/dia, STJ diameter in systole/diastole; AAO sys/dia, AAO diameter in systole/diastole; DAO sys/dia, DAO diameter in systole/ diastole; ICC, intraclass correlation coefficient. Other abbreviations as in Table 1.

\section{Reliability of Aortic Measurements}

Intra- and interobserver reproducibilities in the analysis of AOs and AOd at the 4 levels of the aorta were confirmed in 10 randomly selected participants by means of Bland-Altman analysis and ICC. Measurements of aortic diameters proved to be highly reproducible. Figure 4 shows the Bland-Altman plots for interobserver variability (bias \pm limits of agreement), while Table 4 summarizes the intra- and interobserver reproducibilities from the Bland-Altman analysis and ICC.

\section{Discussion}

To our knowledge, this is the first report to demonstrate that: (1) children with MFS or LDS may already have decreased aortic elasticity even before AoR dilatation progresses, and (2) decreased aortic distensibility and CTD type are independent determinant factors of aortic dilatation in children with MFS or LDS. In agreement with previous reports, ${ }^{9-11}$ the segmental aortic elasticity parameters in MFS were significantly lower than in controls. Moreover, we demonstrated that aortic elasticity had already become reduced in children with MFS or LDS without AoR dilatation. An earlier study found that adult MFS patients with non-dilated AoR had increased pulse wave velocity because of diminished aortic elasticity, ${ }^{22}$ but did not describe impaired aortic elasticity in children with MFS or LDS in the early stage of disease. As aortic stiffness is related to disruption of the medial elastic fibers, ${ }^{6,7}$ it may represent a good marker of aortic medial pathology in both CTD patients and children with congenital heart disease. ${ }^{21,23}$ Prakash et al reported that in an MRI-based study reduced aortic strain was independently associated with higher rates of surgical aortic replacement and AoR dilation in relatively young patients (mean age: 24 years) with CTDs. ${ }^{9}$ Similar findings were presented by Nollen et al in adult MFS patients. ${ }^{8}$ Both of those studies supported the utility of measuring aortic elastic properties to manage adults and young adults with CTDs.

With regard to the treatment of MFS and LDS, atenolol and/or losartan have shown promise in reducing aortic stiffness. ${ }^{24-27}$ However, there is no consensus on when these therapies should be started in children with CTDs, especially in those with non-dilated AoR. A recent prospective randomized study showed that both atenolol and losartan reduced the rate of aortic dilatation in MFS children. ${ }^{28}$ Interestingly, these medications were more effective on improving AoR dilatation in children who started treatment at a younger age. Based on our data and the previous literature, we propose that these therapies be prescribed earlier for children with MFS or LDS even before AoR dilatation has progressed, because aortic stiffness may have already increased and contribute to medication resistance in the later stages.

Besides increased aortic stiffness, the type of CTD was an independent determinant factor of aortic dilatation in this study. We speculate that this result can be attributed to the histological characteristics of LDS, in which more severe medial elastic fiber disruption of the aortic wall leads to more rapid progression of aortic dilatation as compared with MFS. ${ }^{2}$

BP levels were similar among the groups in this study despite higher aortic stiffness in the patients' groups. There are contradictory results on the association of BP level and aortic stiffness in MFS. Teixido-Tura et al have reported that adult MFS patients with increased aortic stiffness showed higher BP than controls, ${ }^{29}$ whereas Hirata et al have showed no significant difference in BP between MFS and controls despite higher aortic stiffness in the MFS patients. ${ }^{10} \mathrm{We}$ speculate this discrepancy might reflect the different stages in the disruption of the medial elastic fibers. Increased aortic stiffness by disrupting elastin in the aortic wall is associated with subsequent development of hypertension. ${ }^{30,31}$ Several clinical studies have showed that increased aortic stiffness in normotensive individuals is associated with subsequent $\mathrm{BP}$ progression. ${ }^{32,33}$ Children with MFS or LDS who have increased aortic stiffness may develop hypertension in adulthood if the disruption of the medial elastic fibers progresses.

Measurement of echocardiologic parameters to assess the elastic properties of the aorta, including distensibility, strain, and stiffness index, is a simple and repeatable method that can be incorporated into routine echocardiography. This noninvasive approach has been used for patients of various ages and diseases to evaluate aortic stiffness, ${ }^{15,18,19}$ and has been validated in coronary artery disease through comparisons with invasive methods. ${ }^{34}$ Although MRI is regularly used to assess aortic elasticity in CTD patients, routine testing can become expensive, time consuming, and traumatic for small children. In general, echocardiography is performed more frequently than MRI on patients with CTDs to follow aortic changes. Our current results confirmed that the measurement of aortic elasticity using echocardiography is useful and reliable in evaluating aortic impairment in children with CTDs.

\section{Study Limitations}

First, it was retrospective, cross-sectional, and included a small sample. Second, we were not blinded to the patients' diagnoses. Third, we performed noninvasive brachial pressure measurement with a sphygmomanometer to determine aortic pressure 
instead of direct measurement, although this method has been well correlated for distensibility of the AAO. ${ }^{34}$

As far as we know, segmental aortic stiffness and its relation to aortic dilatation using echocardiography in children with MFS or LDS has not been described. Thus, this is the first report revealing that children with MFS or LDS with a non-dilated AoR already have decreased aortic elasticity, knowledge that may assist in the management of vascular complications in children with these CTDs.

In conclusion, the present study demonstrated that aortic stiffness as evaluated by echocardiography was increased in children afflicted by MFS or LDS, even before AoR dilatation progresses.

\section{Financial Support Statement / Disclosure}

The authors have no conflicts of interest or financial relationships relevant to this article to disclose.

\section{References}

1. Marsalese DL, Moodie DS, Vacante M, Lytle BW, Gill CC, Sterba $\mathrm{R}$, et al. Marfan's syndrome: Natural history and long-term followup of cardiovascular involvement. J Am Coll Cardiol 1989; 14: $422-431$.

2. Loeys BL, Schwarze U, Holm T, Callewaert BL, Thomas GH, Pannu $\mathrm{H}$, et al. Aneurysm syndromes caused by mutations in the TGF-beta receptor. N Engl J Med 2006; 355: 788-798.

3. Jondeau G, Detaint D, Tubach F, Arnoult F, Milleron O, Raoux F, et al. Aortic event rate in the Marfan population: A cohort study. Circulation 2012; 125: 226-232.

4. Meijboom LJ, Timmermans J, Zwinderman AH, Engelfriet PM, Mulder BJ. Aortic root growth in men and women with the Marfan's syndrome. Am J Cardiol 2005; 96: 1441 - 1444.

5. Maleszewski JJ, Miller DV, Lu J, Dietz HC, Halushka MK. Histopathologic findings in ascending aortas from individuals with LoeysDietz syndrome (LDS). Am J Surg Pathol 2009; 33: 194-201.

6. Okamoto RJ, Xu H, Kouchoukos NT, Moon MR, Sundt TM 3rd. The influence of mechanical properties on wall stress and distensibility of the dilated ascending aorta. J Thorac Cardiovasc Surg 2003; 126: $842-850$.

7. Marque V, Kieffer P, Gayraud B, Lartaud-Idjouadiene I, Ramirez F, Atkinson J. Aortic wall mechanics and composition in a transgenic mouse model of Marfan syndrome. Arterioscler Thromb Vasc Biol 2001; 21: 1184-1189.

8. Nollen GJ, Groenink M, Tijssen JG, Van Der Wall EE, Mulder BJ. Aortic stiffness and diameter predict progressive aortic dilatation in patients with Marfan syndrome. Eur Heart J 2004; 25: 1146-1152.

9. Prakash A, Adlakha H, Rabideau N, Hass CJ, Morris SA, Geva T, et al. Segmental aortic stiffness in children and young adults with connective tissue disorders: Relationships with age, aortic size, rate of dilation, and surgical root replacement. Circulation 2015; 132: 595-602.

10. Hirata K, Triposkiadis F, Sparks E, Bowen J, Wooley CF, Boudoulas $\mathrm{H}$. The Marfan syndrome: Abnormal aortic elastic properties. $J$ Am Coll Cardiol 1991; 18: 57-63.

11. Baumgartner D, Baumgartner C, Mátyás G, Steinmann B, LöfflerRagg J, Schermer E, et al. Diagnostic power of aortic elastic properties in young patients with Marfan syndrome. J Thorac Cardiovasc Surg 2005; 129: 730-739.

12. Singh MN, Lacro RV. Recent clinical drug trials evidence in Marfan syndrome and clinical implications. Can J Cardiol 2016; 32: 66-77.

13. Loeys BL, Dietz HC, Braverman AC, Callewaert BL, De Backer J, Devereux RB, et al. The revised Ghent nosology for the Marfan syndrome. J Med Genet 2010; 47: 476-485.

14. Lopez L, Colan SD, Frommelt PC, Ensing GJ, Kendall K, Younoszai $\mathrm{AK}$, et al. Recommendations for quantification methods during the performance of a pediatric echocardiogram: A report from the Pediatric Measurements Writing Group of the American Society of Echocardiography Pediatric and Congenital Heart Disease Council. J Am
Soc Echocardiogr 2010; 23: 465-495.

15. Oyamada J, Toyono M, Shimada S, Aoki-Okazaki M, Takahashi T. Altered central aortic elastic properties in Kawasaki disease are related to changes in left ventricular geometry and coronary artery aneurysm formation. J Am Soc Echocardiogr 2012; 25: 690-696.

16. Pettersen MD, Du W, Skeens ME, Humes RA. Regression equations for calculation of $\mathrm{z}$ scores of cardiac structures in a large cohort of healthy infants, children, and adolescents: An echocardiographic study. J Am Soc Echocardiogr 2008; 21: 922-934.

17. Gautier M, Detaint D, Fermanian C, Aegerter P, Delorme G, Arnoult $F$, et al. Nomograms for aortic root diameters in children using twodimensional echocardiography. Am J Cardiol 2010; 105: 888-894.

18. Oulego-Erroz I, Alonso-Quintela P, Mora-Matilla M, Gautreaux Minaya S, Lapeña-López de Armentia S. Ascending aorta elasticity in children with isolated bicuspid aortic valve. Int J Cardiol 2013; 168: $1143-1146$.

19. Weismann CG, Lombardi KC, Grell BS, Northrup V, Sugeng L. Aortic stiffness and left ventricular diastolic function in children with well-functioning bicuspid aortic valves. Eur Heart J Cardiovasc Imaging 2016; 17: 225-230.

20. Nakagawa R, Kuwata S, Kurishima C, Saiki H, Iwamoto Y, Sugimoto M, et al. Arterial stiffness in patients after Kawasaki disease without coronary artery involvement: Assessment by performing brachial ankle pulse wave velocity and cardio-ankle vascular index. J Cardiol 2015; 66: 130-134.

21. Kojima T, Kuwata S, Kurishima C, Iwamoto Y, Saiki H, Ishido H, et al. Aortic root dilatation and aortic stiffness in patients with single ventricular circulation. Circ J 2014; 78: 2507-2511.

22. Kiotsekoglou A, Moggridge JC, Saha SK, Kapetanakis V, Govindan $\mathrm{M}$, Alpendurada F, et al. Assessment of aortic stiffness in Marfan syndrome using two-dimensional and Doppler echocardiography. Echocardiography 2011; 28: 29-37.

23. Seki M, Kurishima C, Saiki H, Masutani S, Arakawa H, Tamura M, et al. Progressive aortic dilation and aortic stiffness in children with repaired tetralogy of Fallot. Heart Vessels 2014; 29: 83-87.

24. Rios AS, Silber EN, Bavishi N, Varga P, Burton BK, Clark WA, et al. Effect of long-term beta-blockade on aortic root compliance in patients with Marfan syndrome. Am Heart J 1999; 137:1057-1061.

25. Groenink M, de Roos A, Mulder BJ, Spaan JA, van der Wall EE. Changes in aortic distensibility and pulse wave velocity assessed with magnetic resonance imaging following beta-blocker therapy in the Marfan syndrome. Am J Cardiol 1998; 82: 203-208.

26. Sandor GG, Alghamdi MH, Raffin LA, Potts MT, Williams LD, Potts JE, et al. A randomized, double blind pilot study to assess the effects of losartan vs. atenolol on the biophysical properties of the aorta in patients with Marfan and Loeys-Dietz syndromes. Int $J$ Cardiol 2015; 179: 470-475.

27. Bhatt AB, Buck JS, Zuflacht JP, Milian J, Kadivar S, Gauvreau K, et al. Distinct effects of losartan and atenolol on vascular stiffness in Marfan syndrome. Vasc Med 2015; 20: 317-325.

28. Lacro RV, Dietz HC, Sleeper LA, Yetman AT, Bradley TJ, Colan $\mathrm{SD}$, et al. Atenolol versus losartan in children and young adults with Marfan's syndrome. N Engl J Med 2014; 371: 2061-2071.

29. Teixido-Tura G, Redheuil A, Rodríguez-Palomares J, Gutiérrez L, Sánchez V, Forteza A, et al. Aortic biomechanics by magnetic resonance: Early markers of aortic disease in Marfan syndrome regardless of aortic dilatation? Int J Cardiol 2014; 171: 56-61.

30. Mitchell GF. Arterial stiffness and hypertension: Chicken or egg? Hypertension 2014; 64: 210-214.

31. Salaymeh KJ, Banerjee A. Evaluation of arterial stiffness in children with Williams syndrome: Does it play a role in evolving hypertension? Am Heart J 2001; 142: 549-555.

32. Dernellis J, Panaretou M. Aortic stiffness is an independent predictor of progression to hypertension in nonhypertensive subjects. Hypertension 2005; 45: 426-431.

33. Kaess BM, Rong J, Larson MG, Hamburg NM, Vita JA, Levy D, et al. Aortic stiffness, blood pressure progression, and incident hypertension. JAMA 2012; 308: 875-881.

34. Stefanadis C, Stratos C, Boudoulas H, Kourouklis C, Toutouzas P. Distensibility of the ascending aorta: Comparison of invasive and non-invasive techniques in healthy men and in men with coronary artery disease. Eur Heart J 1990; 11: 990-996. 